P046

ENSURING QUALITY-ASSURED AND PERSONALIZED ONLINE SELF-TESTING WITHIN A MARKET-DRIVEN CONTEXT

${ }^{1}$ Koenraad Vermey, ${ }^{2}$ Chantal Den Daas, ${ }^{1}$ Wessel Zweers, ${ }^{3}$ Jan Van Bergen, ${ }^{1}$ Hanna Bos*. ${ }^{1}$ Soa AIDS Nederland, Amsterdam, Netherlands; ${ }^{2}$ National Institute for Public Health and the Environment (RIVM), Epidemiology and Surveillance, Centre for Infectious Diseases Control, Bilthoven, Netherlands; ${ }^{3}$ National Institute for Public Health and the Environment (RIVM), Centre for Infectious Disease Control, Bilthoven, Netherlands

10.1136/sextrans-2019-sti.251

Background The number of private and online providers of STI tests is increasing in the Netherlands. The autonomy and accessibility of online self-testing may contribute to timely diagnosis, lower healthcare costs and shorter waiting lists at STI clinics. But ill-informed self-testing can also lead to underdiagnosis and insufficient partnermanagement of STIs. To improve linkage to high quality private testing providers the online advice application Advies.chat was launched in 2017. We assessed process indicators for the successful implementation of this online advice instrument in 2018.

Methods The application generates tailored advices based on clinical guidelines. The questionnaire takes into account personal characteristics, sexual behaviour, sexual risks and symptoms. The advice refers to STI testing providers if testing is indicated and explains which specific STIs need to be tested for. All test advices refer to GP's and the specific diagnostic tests offered by selected online testing providers. Key populations (MSM, sex workers and young people $<25$ years) are also referred to STI clinics. Anonymous process data from the Advies.chat database were analysed.

Results Advies. chat was visited 337,736 times in 2018; 113,257 visitors started the questionnaire, 17,449 the chatbot. Visits increased on Sundays, peaked on Mondays and decreased during the week. The most indicated reason for using Advies.chat was the 'possibility of being STI or HIV infected' (75\%). Around $60 \%$ finished the questionnaire, leading to 65,736 advices and 8,700 clicks to online self-test providers.

Conclusion Online self-management tools can play a keyrole in improving the quality of the growing online STI testing market. Advies.chat shows that online triage and tailored advice is feasible and increases traffic to quality testing providers. The contribution of Advies.chat to the estimated 430,000 consultations at GP's and STI clinics in the Netherlands is sizable. Methods need to be developed to assess the impact of online self-management and self-testing on STI control.

Disclosure No significant relationships.

\section{P047 THE THREE RS: RECALLS, REMINDERS AND RETESTING FOR CHLAMYDIA - VIEWS OF GPS AND YOUNG ADULTS}

${ }^{1}$ Alaina Vaisey ${ }^{*},{ }^{2}$ Meredith Temple-Smith, ${ }^{3}$ Anna Yeung, ${ }^{2}$ Anna Wood, ${ }^{4}$ Rebecca Lorch, ${ }^{5}$ Rebecca Guy, ${ }^{5}$ Basil Donovan, ${ }^{6}$ Christopher Fairley, ${ }^{1}$ Jane Hocking. ${ }^{1}$ University of Melbourne, Melbourne School of Population and Global Health, Carlton, Australia; ${ }^{2}$ University of Melbourne, Department of General Practice, Carlton, Australia; ${ }^{3}$ St. Michael's Hospital, Centre for Urban Health Solutions, Li Ka Shing Knowledge Institute, Toronto, Canada; ${ }^{4}$ South Eastern Sydney Local Health District, HIV and Related Programs Unit, Sydney, Australia; ${ }^{5}$ University of New South Wales, The Kirby Institute, Sydney, Australia; ${ }^{6}$ Melbourne Sexual Health Centre, Melbourne, Australia

10.1136/sextrans-2019-sti.252
Background Chlamydia reinfection increases the risk of pelvic inflammatory disease. Reinfection is common in Australia and while clinical guidelines recommend retesting 3 months posttreatment, less than $25 \%$ are retested. We aimed to examine general practitioner (GP) and patient views on retesting for chlamydia and recall/reminder systems to facilitate retesting.

Methods As part of a trial of chlamydia testing in general practice, GPs were provided with resources and support to implement recall/reminder systems to increase retesting. GPs' attitudes and practices were examined pre- and mid-intervention using semi-structured interviews. Semi-structured interviews were also conducted with patients throughout the trial. Data were analysed thematically.

Results 44 GPs undertook a pre-intervention and 24 a midintervention interview; 22 patients were interviewed. GPs viewed recalls/reminders as essential to a formal chlamydia control program. There was disparity in whether systems to enable retesting were adopted during the intervention. Barriers to implementing these systems included concerns about costs and time required to 'chase up' patients; these barriers persisted during the intervention. Concerns about privacy were raised by most GPs but not patients. Over half of patients were not provided with advice about retesting at the time of their initial chlamydia test. Of the four patients who tested positive, two were retested as per guidelines. Patients were universally supportive of receiving reminders for chlamydia retesting, though retesting when at the clinic for another reason was viewed as 'more practical'. Patients did not have strong preferences about reminder type (letter, SMS, email). Knowledge gaps were identified by both GPs and patients, and GPs identified a need to improve knowledge of the risks of chlamydia reinfection.

Conclusion GPs raised more concerns about retesting and reminders than patients. Increasing GP and patient knowledge of the risks of reinfection is crucial. GPs require additional support to implement strategies to increase re-testing. Disclosure No significant relationships.

\section{P048 IMPROVING 'HOME-BASED' STI/HIV SELF-SAMPLING AND BOOSTING SAMPLE RETURN RATES}

${ }^{1}$ Paul Flowers*, ${ }^{2}$ Melvina Owusu, ${ }^{3}$ Maria Pothoulaki, ${ }^{2}$ Fiona Mapp, ${ }^{3}$ Gaby Vojt ${ }^{4}$ Catherine Mercer, ${ }^{5}$ Jackie Cassell, ${ }^{4}$ Sonali Wayal, ${ }^{6}$ Merle Symonds, ${ }^{7}$ Rak Nandwani, ${ }^{2}$ Alison Howarth, ${ }^{2}$ John Saunders, ${ }^{8} \mathrm{~S}$ Brice, ${ }^{6}$ Alex Comer -Schwartz, ${ }^{9}$ Claudia Estcourt. ${ }^{1}$ University of Glasgow, MRC/CSO Social and Public Health Sciences Unit, Glasgow, UK; ${ }^{2}$ UCL, London, UK; ${ }^{3}$ Glasgow Caledonian University, Glasgow, UK; ${ }^{4}$ University College London, Institute for Global Health, London, UK; ${ }^{5}$ Brighton and Sussex Medical School, Primary Care and Public Health, Brighton and Hove, UK; ${ }^{6}$ Barts Health NHS Trust, London, UK; ${ }^{7}$ NGS Greater Glasgow and Clyde, Glasgow, UK; ${ }^{8}$ NHS Barts, London, UK; ${ }^{9}$ Glasgow Caledonian University, School of Health and Life Sciences, Glasgow, UK

\subsection{6/sextrans-2019-sti.253}

Background STI/HIV self-sampling has the potential to improve sexual health by increasing access to testing, yet uptake and sample return rates are currently sub-optimal. Our inter-disciplinary research team explored home-based self-sampling from the user perspective, to inform its optimisation (within the context of LUSTRUM, a UK based trial of Accelerated Partner Therapy (APT)). We describe how implementation science approaches were used to reconcile user, professional and practical requirements for APT and to suggest a series of simple improvements. 